\title{
Síndrome BRASH: A propósito de un caso acerca de un fenómeno sinérgico
}

\author{
Alejandro J. Moya-Álvarez ${ }^{1 *}$ y David J. Hernández-Solano ${ }^{2}$ \\ ${ }^{1}$ Medicina de Emergencias, Hospital Dr. Rafael Ángel Calderón Guardia; ${ }^{2}$ Área de salud de Oreamuno, Clínica Dr. Ricardo Jiménez Núñez. Caja \\ Costarricense de Seguro Social, San José, Costa Rica
}

\begin{abstract}
Resumen
El síndrome BRASH (la combinación de bradicardia, insuficiencia renal, fármacos bloqueantes del nodo atrioventricular, choque e hiperpotasemia) es una patología recientemente descrita, la cual se confunde frecuentemente con una bradicardia o hiperpotasemia aislada. Según la literatura, este es uno de los errores más comunes y que mayor impacto negativo ofrece sobre el pronóstico del paciente. La piedra angular de este síndrome es la sinergia entre todos los factores que lo componen, por tanto, la literatura refiere que el clínico debe enfocarse en la identificación y el manejo de todos estos de manera simultánea. El tratamiento recae fundamentalmente en el manejo pronto de la hiperpotasemia, así como la resucitación con fluidos; y en algunas ocasiones se necesitarán métodos más avanzados de manejo, como antídotos para revertir el efecto del betabloqueo o calcioantagonistas, entre otros. Presentamos el caso de un hombre de 84 años que cumple con todos los criterios para el diagnóstico de este síndrome y en el cual el reconocimiento temprano tuvo impacto positivo en la evolución y el pronóstico de este.
\end{abstract}

Palabras clave: Síndrome BRASH. Bradicardia. Hiperpotasemia. Insuficiencia renal. Caso clínico.

\section{BRASH syndrome: A case about a synergic syndrome}

\section{Abstract}

BRASH syndrome is compound by the combination of bradycardia, renal failure, AV node blockers, shock and hyperkalemia. This illness has been recently described and medical reviews have demonstrated that due to its novelty some physicians usually confuse it with an isolated hyperkalemia or bradycardia. The cornerstone of this syndrome is the synergistic phenomenon among all of its components so, the clinician must consider all of this and manage it simultaneously. The treatment must focus principally on the hyperkalemia management as well as an adequate fluid resuscitation. In some cases, the medical team must use some other advanced therapies, as reversal effect antidotes to revert beta blockers and calcium antagonist effects. We present a case of an 84-year-old male with all BRASH syndrome components in which the early recognition of its pathology had a positive impact on his prognosis.

Key words: BRASH syndrome. Bradycardia. Hyperkalemia. Renal failure. Clinical case.

\footnotetext{
Correspondencia:

*Alejandro J. Moya-Álvarez

E-mail: amoya@resusimtation.com

Fecha de aceptaación: 05-05-202

DOI: 10.24875/REIE.21000040

Disponible en internet: 30-07-2021

Rev Educ Investig Emer. 2021;3(2):113-117 www.medicinadeemergencias.com

(1) Es 2021 Sociedad Mexicana de Medicina de Emergencias, AC. Publicado por Permanyer México SA de CV. Este es un artículo open access bajo la licencia CC BY-NC-ND (http://creativecommons.org/licenses/by-nc-nd/4.0/).
} 


\section{Introducción}

El síndrome BRASH (por su acrónimo en inglés) se define como la presentación de un paciente que clínicamente muestra: bradicardia, insuficiencia renal (renal failure), bloqueo del nodo atrioventricular (AV) en el contexto de uso de fármacos tales como betabloqueantes o calcioantagonistas, choque e hiperpotasemia. Este síndrome fue recientemente descrito en el año 2016 por el Dr. Joshua Farkas, profesor asistente de Neumología y Cuidados críticos en la Universidad de Vermont/Burlington en EE.UU. La literatura actual concuerda en que esta patología con frecuencia es subdiagnosticada o confundida con una hiperpotasemia 0 bradicardia aisladas ${ }^{1-3}$.

Está bien establecido que la hiperpotasemia y los medicamentos bloqueadores del nodo AV pueden causar bradicardia. Estudios en animales y múltiples reportes de caso demuestran cómo ambos factores en conjunto producen un fenómeno sinérgico que puede llevar a una bradicardia severa y bloqueos de alto grado en comparación de cuando los mismos se presentan de manera aislada ${ }^{3,4}$.

Se ha observado que la frecuente asociación entre los fármacos antihipertensivos utilizados en la población adulta mayor, así como una prevalencia aumentada de función renal limítrofe o deteriorada en este grupo etario, hacen que su diagnóstico sea más frecuente en esta población. No obstante, también existen reportes en individuos más jóvenes.

\section{Presentación del caso}

Recibimos un hombre de 84 años, residente en la capital, San José, conocido portador de hipertensión arterial riesgo $\mathrm{C}$, cardiopatía isquémica, enfermedad renal crónica en control con nefrología y enfermedad pulmonar obstructiva crónica. Actualmente no requiere de oxigenoterapia domiciliar. Presenta un adecuado control de sus patologías crónicas y actualmente recibe como tratamiento crónico: atenolol $50 \mathrm{mg}$ BID vía oral (VO), enalapril $20 \mathrm{mg} \mathrm{c} / \mathrm{d}$ VO, venlafaxina $75 \mathrm{mg} \mathrm{c} / \mathrm{d}$ VO, furosemida $40 \mathrm{mg} \mathrm{BID} \mathrm{VO}$, lovastatina $40 \mathrm{mg} \mathrm{c} / \mathrm{d}$ VO y loratadina $10 \mathrm{mg} c / \mathrm{d}$ VO. Fue referido desde una clínica periférica en donde ingreso con historia de disnea de tres días de evolución asociado a astenia y adinamia. También sus familiares refieren que desde el día previo a la visita a la clínica «no orina». El día de la consulta presenta deterioro progresivo del estado de consciencia, por lo que deciden buscar atención médica. Una vez en la clínica, y durante el abordaje inicial, se documentan los siguientes signos vitales: presión arterial $85 / 50 \mathrm{mmHg}$, frecuencia cardiaca 33 Ipm, frecuencia respiratoria 26 rpm, saturación de oxígeno $90 \%$ y escala de coma de Glasgow de 9 puntos. Se realiza rápidamente un electrocardiograma (ECG) (Fig. 1). En ese momento se traslada con carácter urgente a nuestro servicio de emergencias (15 minutos de traslado aproximadamente). Una vez en nuestro hospital se repite un nuevo ECG, que muestra similares hallazgos. Además, se realiza un i-STAT ${ }^{\circledR}$ que documenta acidosis metabólica con brecha aniónica aumentada y una acidosis respiratoria concomitante. Debido a los hallazgos anteriormente descritos se plantea la posibilidad de un síndrome BRASH, por lo que se inicia el tratamiento correspondiente basado en hidratación con control clínico, corrección de la hiperpotasemia con medidas de redistribución y de eliminación y finalmente colocación de marcapasos transvenoso. Posteriormente sus estudios de laboratorio muestran un aumento de $4.6 \mathrm{mg} / \mathrm{dl}$ sobre su creatinina basal (6.2 $\mathrm{mg} / \mathrm{dl}$ en su última consulta con nefrología), lo que confirma la reagudización de su lesión renal crónica. Una vez instaurado el tratamiento, nuestro paciente evoluciona satisfactoriamente hasta ser ingresado en el servicio de hospitalización para el control nefrológico correspondiente.

\section{Discusión}

El BRASH es un síndrome que engloba diversos mecanismos patológicos, los cuales en conjunto generan un ciclo vicioso hacia un desenlace fatal si no se trata a tiempo. Este síndrome es comúnmente desencadenado por un factor incitador o gatillo en un paciente con compromiso renal. Debe hacerse hincapié en que estos individuos cuentan con varios factores de riesgo, los cuales generan un círculo vicioso (Fig. 2). Estos factores son: bradicardia, insuficiencia renal, uso de fármacos bloqueantes del nodo $\mathrm{AV}$, choque e hiperpotasemia. La literatura menciona que el porcentaje de pacientes que presentan todos los componentes del BRASH es aún incierto, sobre todo debido al estadio de la enfermedad en que se aborda al paciente, este podría presentar o no estado de choque ${ }^{5}$.

El principal factor desencadenante es la hipovolemia. Esta última en un riñón previamente lesionado irá en mayor detrimento del aporte sanguíneo hacia estos órganos, empeorando aún más la tasa de filtración glomerular (TFG), generando así una concentración sérica mayor de los medicamentos bloqueantes del nodo AV e hiperpotasemia, todos en conjunto llevarán 


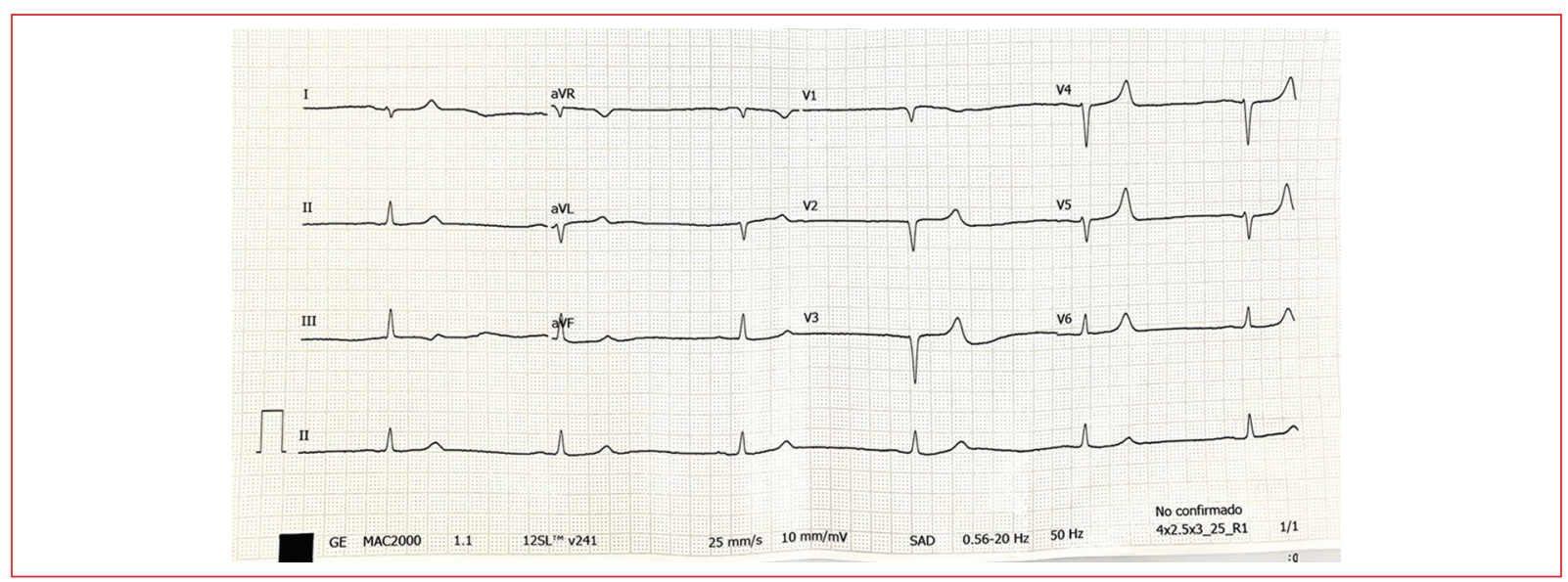

Figura 1. Electrocardiograma del paciente a su ingreso al servicio de emergencias de la clínica periférica. Nótese la bradicardia extrema con bloqueo atrioventricular y ondas T picudas que sugieren las presencia de hiperpotasemia. Además, nótese bradicardia de la unión, frecuencia cardiaca alrededor de 30 lpm, QRS estrecho, ondas t picudas 0 prominentes mayormente visibles desde V3 a V6, sin prolongación del QTc.

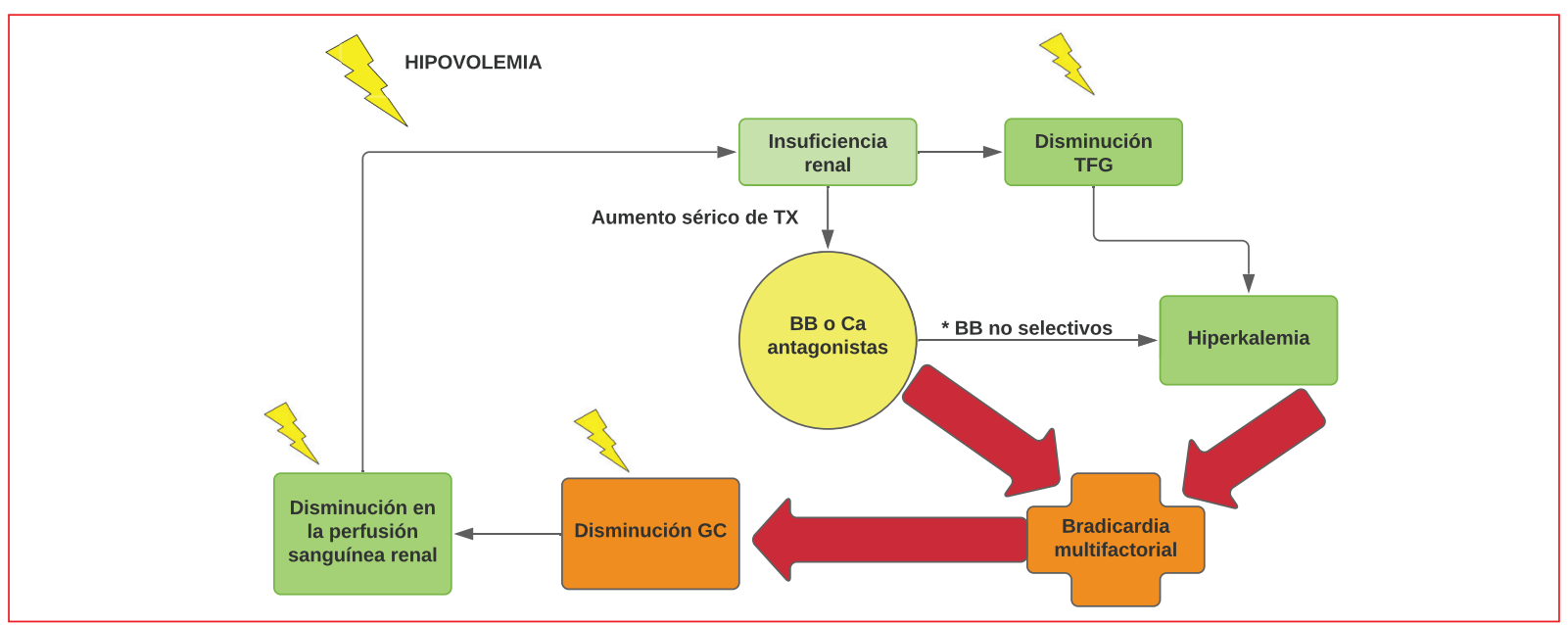

Figura 2. Ciclo vicioso en la etiopatogenia del BRASH (adaptada de Farkas, et al., 20204).

*Los BB no selectivos son causa de hiperpotasemia. Nótese como la hipovolemia se comporta como un gatillo en varios puntos de la cascada fisiopatológica.

TFG: tasa de filtración glomerular; BB: betabloqueadores; GC: gasto cardiaco; Ca: calcio; TX: fármacos.

hacia un bloqueo AV de alto grado y como resultante un bajo gasto cardiaco. Otro factor que no es parte del ciclo, pero debe tenerse en cuenta es el uso concomitante de inhibidores de la enzima convertidora de angiotensina 0 inhibidores de angiotensina, los cuales pueden empeorar la hiperpotasemia ${ }^{1,4,6}$.

Es importante hacer notar que el reconocimiento temprano de esta patología mejora el pronóstico y previene complicaciones y se debe ser cauteloso en reconocerlo y así distinguirlo de una bradicardia o hiperpotasemia aisladas. En la hiperpotasemia pura los niveles de potasio presentan la tendencia a ser mucho mayores para provocar arritmias o bloqueos $\mathrm{AV}$, así como cambios electrocardiográficos. Niveles entre 6.0-6.5 mEq/l típicamente se asocian a ondas $\mathrm{T}$ picudas, mientras que niveles cercanos o mayores a $8.0 \mathrm{mEq} / \mathrm{l}$ se relacionan con bradicardia severa y arritmias malignas tales como fibrilación ventricular 0 taquicardia ventricular ${ }^{1,3,7}$. Por otra parte, se ha observado que en el BRASH niveles séricos de potasio cercanos a $5 \mathrm{mEq} / \mathrm{l}$ son suficientes para generar bradiarritmias. 
Tomando en cuenta lo anteriormente citado, muchos de los clínicos a la hora de abordar al paciente se enfocan en el manejo de la bradicardia y en este apartado es de suma importancia destacar que los algoritmos de soporte cardiovascular avanzado al recomendar atropina como primera opción no funcionan de manera adecuada en el BRASH, ya que, por lo mencionado en la fisiopatología de este, la piedra angular en el tratamiento es el manejo rápido de la hiperpotasemia, la cual de no tratarse rápidamente provocará que otros métodos empleados resulten ineficaces ${ }^{1,4,8}$.

Referente a este aspecto, Aziz, et al. mencionan que los adultos mayores presentan una mayor predisposición para el desarrollo de la hiperpotasemia debido a una reducción en la TFG comúnmente preexistente, así como otras comorbilidades frecuentes en esta población, los cuales son factores que alteran la homeostasis de potasio. Además de tener en consideración que estos individuos presentan un umbral menor para desarrollar arritmias cardiacas ante una hiperpotasemia ${ }^{8}$.

Asimismo, la literatura describe que el error más común a la hora de abordar a un paciente con BRASH es fijarse solamente en un componente, por tanto, la clave para obtener buenos resultados será el manejo simultáneo de todos estos. Frecuentemente el uso de distintas terapias y el conocimiento adecuado de la fisiopatología ayudará a evitar tratamientos más agresivos, como, por ejemplo: marcapasos transvenoso y hemodiálisis, entre otros.

\section{Tratamiento}

Debe tenerse en cuenta el manejo inmediato de la hiperpotasemia. Esta alteración debe tratarse incluso cuando el incremento de potasio sea relativamente leve. La evidencia de ondas $T$ picudas en el electrocardiograma, QRS prolongado, ritmo de la unión, cambios significativos en el intervalo ST y onda $\mathrm{T}$, bradicardia o inestabilidad hemodinámica deben tratarse con calcio intravenoso. El calcio estabiliza la membrana cardiaca, lo cual mejora la frecuencia y el gasto cardiaco. Si el ECG no demuestra ninguna mejoría, dosis repetidas deben ser administradas. Además, si el paciente tiene una vía central y no está en paro cardiorrespiratorio pueden utilizarse $3 \mathrm{~g}$ de gluconato de calcio intravenoso, así como también el cloruro de calcio como alternativa.

Por otra parte, posterior a la estabilización de la membrana cardiaca, otros métodos que resultan eficaces para el manejo de la hiperpotasemia son: la insulina intravenosa junto a dextrosa, lo cual genera homeostasis del potasio al ingresar este ion adonde pertenece, en su gran mayoría al espacio intracelular; el uso de albuterol nebulizado también debe considerarse de no resultar eficaces estos anteriores pasos, ya que también genera beneficios potenciales en términos de hiperpotasemia y bradicardia que se presen$\tan$ en este contexto; otro método recomendado es también el empleo de diuréticos caliuréticos tales como bumetanida o furosemida vía intravenosa, así como también diuréticos tiazídicos.

En cuanto a esta medida debe remarcarse que pacientes con una hiperpotasemia marcada en los cuales no se produce una respuesta adecuada al uso de diuréticos a altas dosis y estabilización hemodinámica, frecuentemente requerirán diálisis urgente como manejo definitivo de la hiperpotasemia. No obstante, estos medicamentos no deben emplearse de manera sistemática, debe tenerse en cuenta que estos individuos comúnmente presentan hipoperfusión generalizada debida al bajo gasto cardiaco y a la hipovolemia. Por tanto, antes de su uso debe evaluarse la resucitación adecuada del paciente con fluidoterapia. Si está presente la hipovolemia debe manejarse tan pronto como se reconozca ${ }^{1,3}$.

En cuanto a la fluidoterapia, debe reconocerse que el estado volumétrico varía ampliamente entre los distintos individuos con BRASH, no obstante, se puede rescatar que los distintos autores mencionan que la mayoría se encuentran hipovolémicos al momento del diagnóstico. Por tanto, algunos pacientes progresan a una insuficiencia renal oligúrica o anúrica y consecuentemente a retención de líquidos y un estado de sobrecarga de volumen.

Pacientes con acidosis urémica e hiperpotasemia comúnmente presentan mejoría con la administración de bicarbonato de sodio $(150 \mathrm{mEq} / \mathrm{l}$ diluido en 1 I de solución glucosada al $5 \%$ ), lo anterior haciendo énfasis en que al administrar 1 I de esta solución glucosada compuesta, mayor cantidad de esta se quedará en el espacio intravascular, mejorando así la perfusión renal y el cuadro de acidosis 4 .

Otro aspecto importante es interrogar al paciente (o familiares) acerca del uso de fármacos que intervienen en la conducción AV. En tal caso, el uso de betaagonistas como el isoproterenol son la opción preferida en pacientes que no están en estado de choque. En determinado caso la primera elección será el uso de epinefrina para estabilizar al paciente y posteriormente realizar el traslape a isoproterenol. Las dosis de estos fármacos deben titularse para llevar a una frecuencia cardiaca entre 60-80 Ipm. 
El uso de epinefrina en pacientes con insuficiencia renal ha sido cuestionado por algunos autores, sobre todo debido a la depleción de la enzima renalasa, la cual se encuentra disminuida en estos pacientes. Esta es la encargada de metabolizar la epinefrina. Además, niveles séricos elevados de dicha hormona generan vasoconstricción renal, lo cual podría agravar la isquemia renal cuando se utiliza de manera prolongada. En contraste, el isoproterenol debido a su pobre acción sobre los receptores alfa presenta un menor efecto de vasoconstricción y, por ende, menor riesgo de isquemia renal ${ }^{5}$.

Asimismo, si estas medidas no resultan eficaces para producir una frecuencia cardiaca meta y el paciente entre su historial de tratamiento utiliza betabloqueadores o calcioantagonistas debe intentarse revertir la toxicidad por estos fármacos con emulsión lipídica, glucagón o infusión de insulina a altas dosis. Finalmente, si no se logra el objetivo meta con todas las anteriores medidas debe recurrirse a técnicas más invasivas, como la instalación de un marcapasos transvenoso.

El síndrome BRASH es una patología frecuente en la población de adultos mayores que presentan factores de riesgo para el desarrollo de este, sin embargo, este síndrome también ha sido descrito en poblaciones más jóvenes. Tener un alto índice de sospecha y juntar todas las piezas de este rompecabezas ayudará a realizar un diagnóstico oportuno y un tratamiento temprano dirigido según las metas establecidas.

\section{Financiamiento}

La presente investigación no ha recibido ninguna beca específica de agencias de los sectores públicos, comercial o sin ánimo de lucro.

\section{Conflicto de intereses}

Los autores declaran no tener conflicto de intereses.

\section{Responsabilidades éticas}

Protección de personas y animales. Los autores declaran que para esta investigación no se han realizado experimentos en seres humanos ni en animales.

Confidencialidad de los datos. Los autores declaran que han seguido los protocolos de su centro de trabajo sobre la publicación de datos de pacientes.

Derecho a la privacidad y consentimiento informado. Los autores han obtenido el consentimiento informado de los pacientes y/o sujetos referidos en el artículo.

\section{Bibliografía}

1. Arif AW, Khan MS, Masri A, Mba B, Talha Ayub M, Doukky R. BRASH syndrome with hyperkalemia: An under-recognized clinical condition. Methodist Debakey Cardiovasc J. 2020;16(3):241-4.

2. Toba N, Calderon A, Erazo C. Síndrome de BRASH: bradicardia, falla renal, bloqueo auriculoventricular, shock e hiperpotasemia. Reporte de un caso. Ciencia Digital. 2018;2:567-80.

3. Grigorov MV, Belur AD, Otero D, Chaudhary S, Grigorov E, Ghafghazi S. The BRASH syndrome, a synergistic arrhythmia phenomenon. Proc (Bayl Univ Med Cent). 2020;33(4):668-70.

4. Farkas JD, Long B, Koyfman A, Menson K. BRASH syndrome: Bradycardia, renal failure, AV blockade, shock, and hyperkalemia. J Emerg Med. 2020;59(2):216-23.

5. Vishnu V, Jamshed N, Amrithanand VT, Thandar S. BRASH syndrome: A case report. J Emerg Med. 2021 Feb 24:S0736-4679(21)00089-5. doi: 10.1016/j.jemermed.2021.01.033. Online ahead of print.

6. Srivastava S, Kemnic T, Hildebrandt KR. BRASH syndrome. BMJ Case Rep. 2020;13(2):e233825.

7. Simmons T, Blazar E. Synergistic bradycardia from beta blockers, hyperkalemia, and renal failure. J Emerg Med. 2019;57(2):e41-4.

8. Aziz EF, Javed F, Korniyenko A, Pratap B, Cordova JP, Alviar CL, et al. Mild hyperkalemia and low eGFR a tedious recipe for cardiac disaster in the elderly: an unusual reversible cause of syncope and heart block. Heart Int. 2011;6(2):e12. 